\title{
In Situ High-Resolution Transmission Electron Microscopy of Structures and Conductance of Silver Nanocontacts*
}

\author{
Hideki Masuda, Kosuke Monna, Tomoko Matsuda, and Tokushi Kizuka ${ }^{\dagger}$ \\ Institute of Materials Science, University of Tsukuba, Tsukuba, Ibaraki 305-8573, Japan
}

(Received 10 November 2008; Accepted 10 February 2009; Published 25 April 2009)

\begin{abstract}
The breaking process of silver nanocontacts (NCs) was observed in situ at room temperature by high-resolution transmission electron microscopy. Simultaneously, the conductance of the NCs was measured. During breaking, the conductance showed a stepwise variation and the step height corresponded to integer multiples of $2 e^{2} / h$, i.e. the quantized conductance levels. Using a conductance feedback control, the NCs exhibiting a certain conductance were observed continuously. The relationship between the structure and conductance of the NCs was investigated; the conductance at a quantized level was associated with NCs with several types of atomistic configurations. [DOI: 10.1380/ejssnt.2009.549]
\end{abstract}

Keywords: Quantized Conductance; Transmission Electron Microscopy; Silver

\section{INTRODUCTION}

Nanocontacts (NCs) composed of metallic elements have been studied in relation to the quantized conductance defined by a quantum $\left(G_{0}=2 e^{2} / h\right.$, where $e$ is the electron charge and $h$ is Planck's constant) [1-18]. For the NCs of noble metals (e.g., copper, silver (Ag) and gold), conductance decreases in a stepwise variation during breaking, and the conductance level at each step height corresponds to approximately integer multiples of $G_{0}[2,4,5]$. According to Sharvin's equation, for a conductance level of approximately $1 G_{0}$, the crosssectional area of the contact is expected as that of a single atom $[2,4,5,16]$. Molecular dynamics and ab initio calculations have shown a similar relationship between the conductance and cross-sectional area $[9,19-22]$.

Recently, however, a classical molecular dynamics simulation coupled with conductance calculations based on a tight binding model shows that the width of the minimum cross-sectional area of the Ag NCs exhibiting $1 G_{0}$ ranges from 0.17 to $0.47 \mathrm{~nm}$, which is comparable to the width of 1 to 2 atoms [23]. Experimentally, the quantization of conductance has been illustrated by mechanically controllable break junction (MCBJ) techniques and nanotip contact techniques based on scanning tunneling microscopy (STM) [24-27]. Rodrigues et al. performed high-resolution transmission electron microscopy (HRTEM) of Ag nanowires prepared by dual-holes drilling using electron beams. They also measured the conductance by a MCBJ technique, unconnected to HRTEM observation [25]. In situ HRTEM with a combination of nanotip manipulation enables us to observe directly the relationship between the atomic configuration and conductance of nanocontacts during breaking [17, 28-33]. In this study, we focused on Ag NCs and used this method. In particular, the NCs were controlled to maintain a certain conductance using a feedback system.

* This paper was presented at International Symposium on Surface Science and Nanotechnology (ISSS-5), Waseda University, Japan, 9-13 November, 2008.

†Corresponding author: kizuka@ims.tsukuba.ac.jp

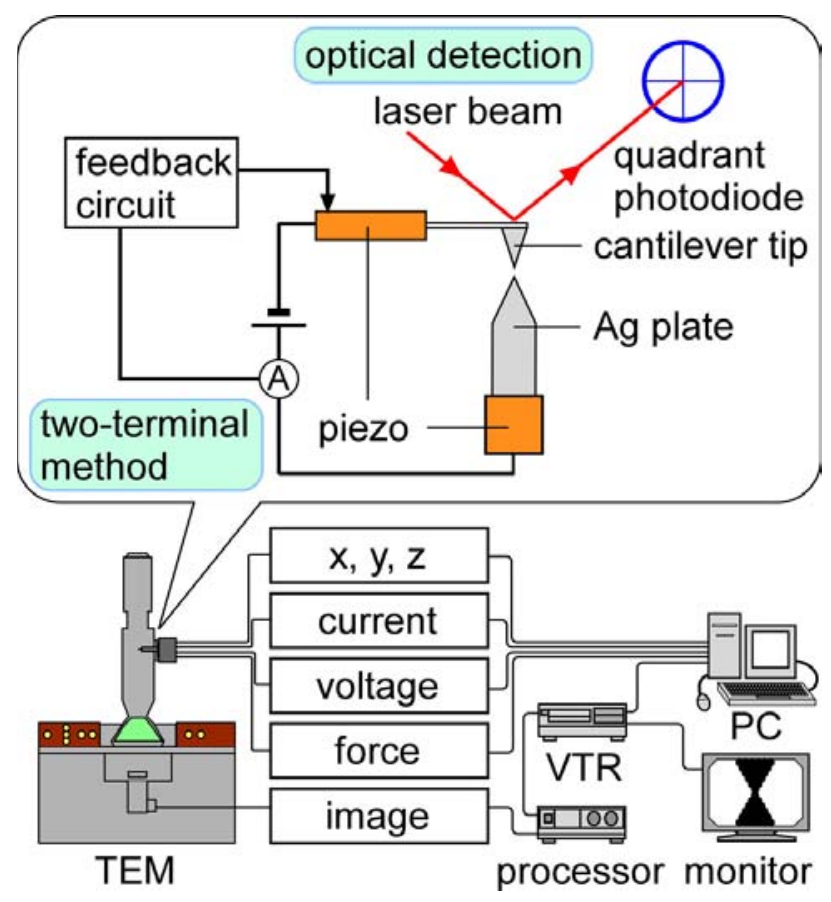

FIG. 1: Illustration of in situ transmission electron microscopy in this study. The voltages applied on piezoelements for specimen displacement along the $x, y, z$ directions, the current through and bias voltage applied to nanocontacts, the force acting on nanocontacts, and images were recorded using a computer.

\section{EXPERIMENTAL}

The experimental method in this study was developed on the basis of in situ HRTEM combined electronic conductance measurement used in STM $[28,32]$. The system is illustrated in Fig. 1. First, we prepared nanometersized Ag tips: Ag was evaporated in a vacuum chamber and deposited on a Si cantilever with a nanotip used in atomic force microscopy. The cantilever tip was attached to the front of a tube piezoelement on a cantilever holder for HRTEM. A Ag plate of $0.2 \mathrm{~mm}$ thickness was attached to the second plate holder. The contact edge of 

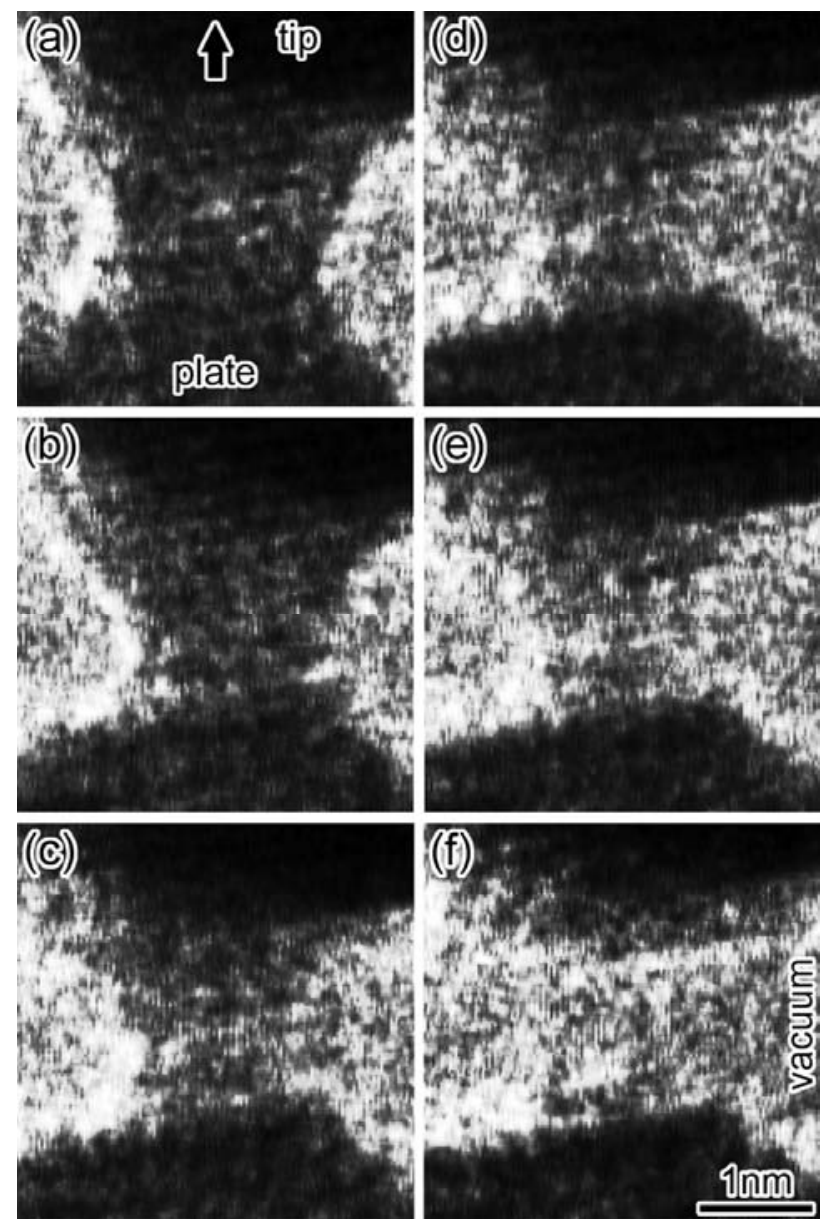

FIG. 2: Time-sequence series of high-resolution images of breaking process of $\mathrm{Ag}$ nanocontact.

the plate for contact was thinned to 5-20 nm by argon ion milling. The cantilever and plate holders were then inserted into the in situ transmission electron microscope at the University of Tsukuba. The specimen chamber of the microscope was evacuated first by a turbomolecular pump and then by an ion pump, resulting in a vacuum of $1 \times 10^{-5} \mathrm{~Pa}$. The cantilever tip was brought into contact with the edge surface of the opposing plate by piezomanipulation while applying a bias voltage of $52 \mathrm{mV}$. The cantilever tip was then pressed into the plate to prepare NCs and then retracted to elongate them. In addition to this simple contact-retraction manipulation, we controlled the distance between the cantilever tip and the plate using a conductance feedback circuit, as a result of which the NCs exhibiting a certain conductance were observed continuously. A series of these manipulations was performed at room temperature. The structural dynamics during the process was observed in situ by lattice imaging by HRTEM using a television capture system. The time resolution of image observations was $17 \mathrm{~ms}$. The force acting on the NCs was simultaneously measured by the optical detection of cantilever deflection. The electrical conductance was measured using a two-terminal method. The high-resolution images and detected signals in this system were simultaneously recorded and analyzed for every image.

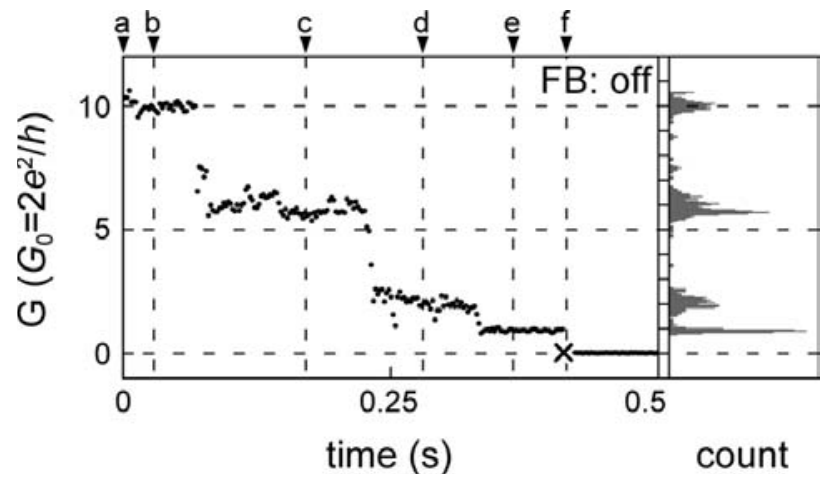

FIG. 3: Variations in conductance of Ag nanocontact during breaking process with a constant retraction speed as function of time. (No feedback control was performed.) The histogram of measured points is inserted in the right side.

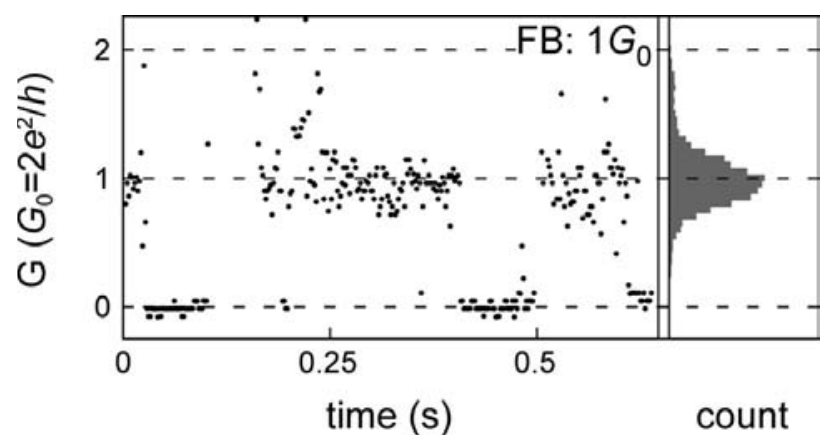

FIG. 4: Variations in conductance of $\mathrm{Ag}$ nanocontact during conductance feedback control to $1 G_{0}$. The histogram of measured points is inserted in the right side.

\section{RESULTS AND DISCUSSIONS}

Figure 2 shows a time-sequence series of high-resolution images of a breaking process of a $\mathrm{Ag} \mathrm{NC}$. The tip and the plate were observed as dark areas, as shown in the upper and the lower sides of Figs. 2(a)-2(f), respectively. The plate was negatively biased by $52 \mathrm{mV}$ to the tip. The bias voltage was much lower than critical voltage for electromigration [26]. In the middle of Fig. 2(a), the contact of approximately $1.5 \mathrm{~nm}$ width is located. On the surfaces of both the tip and the plate, neither contamination nor an oxide layer is observed throughout Figs. 2(a)-2(f). As the tip was retracted from the plate along the direction indicated by the arrow in Fig. 2(a), the NC was thinned gradually, as shown in Figs. 2(a)-2(e). The contact width decreased from 7 to $6,4,2$, and 1 atom-width, as shown in Figs. 2(b)-(e), respectively. Then, the contact broke as shown in Fig. 2(f).

Figure 3 shows variations in the conductance of the $\mathrm{Ag}$ $\mathrm{NC}$ during the same breaking process presented in Fig. 2 as a function of time. Times a-f in Fig. 3 correspond to the times of the observed images in Figs. 2(a)-2(f). During breaking, as time passed, the conductance decreased in a stepwise variation. The histogram of measured points is inserted in the right side of Fig. 3. In this process, peaks were observed at $1,2,6$, and $10 G_{0}$. We repeated at least 

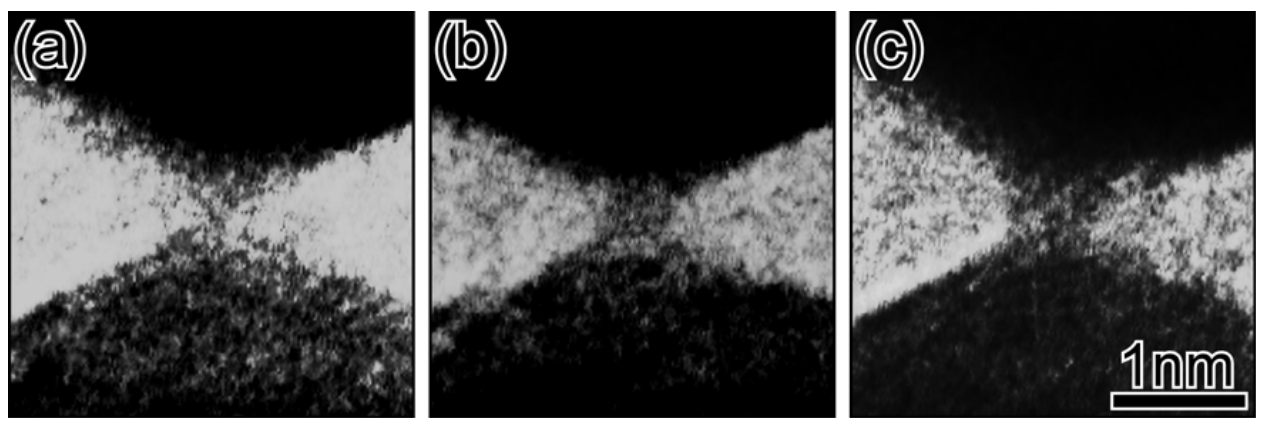

FIG. 5: (a)-(c) High-resolution images of Ag nanocontacts during conductance feedback control to $1 G_{0}$. The minimum crosssectional width in (a), (b), and (c) is 1,2 , and 3 atom-width, respectively.

several hundreds breaking processes, as a result of which peaks at integer multiples of $G_{0}$ were observed, similar to the result for Ag NCs obtained by Ludoph et al. [34]. On the other hand, Rodrigues et al. observed remarkable peak intensity at $2.4 G_{0}$ in a conductance histogram for $\mathrm{Ag}$ NCs [25]. In this study, the intensity at $2.4 G_{0}$ was much lower than that observed by Rodrigues et al.

Figure 4 shows variations in the conductance of a $\mathrm{Ag}$ $\mathrm{NC}$ during the conductance feedback control to $1 G_{0}$ as a function of time. The histogram of measured points is inserted in the right side of Fig. 4. The conductance converged at $1 G_{0}$ repeatedly, and a peak in the histogram corresponded to the setup value. Thus, it is found that the present feedback control functions well. By using this feedback control, the NCs exhibiting the setup conductance was observed continuously.

During the conductance feedback control to $1 G_{0}$, three types of NCs were observed. Figures 5(a)-5(c) show the high-resolution images of such NCs. To measure the width of the minimum cross-sectional area of each image, we measured line intensity at the constriction part of NCs. The minimum cross-sectional width seen in Figs. 5(a)-5(c) corresponded to 1, 2, and 3 atoms, respectively.

For gold NCs, the $1 G_{0}$ state has been related to single atom wires $[19,20]$. On the other hand, it has been pointed out that the conductance of NCs is affected by their atomic configuration [13, 15, 21, 35] and remarkable quantization of conductance is not observed in certain configuration [13]. According to recent theoretical study by Pauly et al., several structures cause a same conductance [23]. Thus, the present observation provides experimental evidence that the $1 G_{0}$ is caused by the three types of the NCs.

\section{CONCLUSIONS}

The relationship between the structure and conductance of Ag NCs was investigated by in situ TEM. Using a conductance feedback control, we observed NCs exhibiting a certain conductance continuously. It was found that the $1 G_{0}$ state is attributed to the NCs with three types of the minimum cross-sectional width.

\section{Acknowledgments}

This work was partly supported by funds for the special Research Project on Nanoscience, the University Research Projects of the University of Tsukuba, the Advanced Processing Mechanical Techniques Foundation, The Ogasawara Foundation for the Promotion of Science and Engineering, Shimadzu Science Foundation, and Grants-in-Aids from the Ministry of Education, Culture, Sports, Science and Technology, Japan (Nos. 18310075 and 19651047).
[1] C. J. Muller, J. M. van Ruitenbeek, and L. J. de Jongh, Phys. Rev. Lett. 69, 140 (1992).

[2] N. Agraï, J. G. Rodrigo, and S. Vieira, Phys. Rev. B 47, 12345 (1993).

[3] J. M. Krans, C. J. Muller, I. K. Yanson, T. C. M. Govaert, R. Hesper, and J. M. van Ruitenbeek, Phys. Rev. B 48, 14721 (1993).

[4] J. I. Pascual, J. Méndez, J. Gómez-Herrero, A. M. Baró, N. García, and V. T. Binh, Phys. Rev. Lett. 71, 1852 (1993).

[5] G. Rubio, N. Agraït, and S. Vieira, Phys. Rev. Lett. 76, 2302 (1996).

[6] J. L. Costa-Krämer, N. García, P. García-Mochales, P. A. Serena, M. I. Marqués, and A. Correia, Phys. Rev. B 55, 5416 (1997).
[7] E. Scheer, N. Agraït, J. C. Cuevas, A. L. Yeyati, B. Ludoph, A. Martín-Rodero, G. R. Bollinger, J. M. Ruitenbeek, and C. Urbina, Nature 394, 154 (1998).

[8] A. I. Yanson, G. R. Bollinger, H. E. van den Brom, N. Agraï, and J. M. van Ruitenbeek, Nature 395, 783 (1998).

[9] S. K. Nielsen, M. Brandbyge, K. Hansen, K. Stokbro, J. M. van Ruitenbeek, and F. Besenbacher, Phys. Rev. Lett. 89, 066804 (2002).

[10] C. Untiedt, A. I. Yanson, R. Grande, G. Rubio-Bollinger, N. Agraït, S. Vieira, and J. M. van Ruitenbeek, Phys. Rev. B 66, 085418 (2002).

[11] U. Landman, W. D. Luedtke, N. A. Burnham, and R. Colton, Science 248, 454 (1990).

[12] M. Brandbyge, J. Schiøtz, M. R. Sørensen, P. Stoltze, K. 
W. Jacobsen, J. K. Nørskov, L. Olesen, E. Laegsgaard, I. Stensgaard, and F. Besenbacher, Phys. Rev. B 52, 8499 (1995).

[13] A. M. Bratkovsky, A. P. Sutton, and T. N. Todorov, Phys. Rev. B 52, 5036 (1995).

[14] U. Landman, W. D. Luedtke, B. E. Salisbury, and R. L. Whetten, Phys. Rev. Lett. 77, 1362 (1996).

[15] A. G. Scherbakov, E. N. Bogachek, and U. Landman, Phys. Rev. B 53, 4054 (1996).

[16] A. Stalder and U. Dürig, J. Vac. Sci. Technol. B 14, 1259 (1996).

[17] A. Correia and N. García, Phys. Rev. B 55, 6689 (1997).

[18] H. Yasuda and A. Sakai, Phys. Rev. B 56, 1069 (1997).

[19] M. R. Sørensen, M. Brandbyge, and K. W. Jacobsen, Phys. Rev. B 57, 3283 (1998).

[20] M. Brandbyge, N. Kobayashi, and M. Tsukada, Phys. Rev. B 60, 17064 (1999).

[21] M. Okamoto and K. Takayanagi, Phys. Rev. B 60, 7808 (1999).

[22] S. R. Bahn, N. Lopez, J. K. Nørskov, and W. Jacobson, Phys. Rev. B 66, 081405 (2002).

[23] F. Pauly, M. Dreher, J. K. Viljas, M. Häfner, J. C. Cuevas, and P. Nielaba, Phys. Rev. B 74, 235106 (2006).

[24] K. Hansen, E. Lægsgaard, I. Stensgaard, and F. Besen- bacher, Phys. Rev. B 56, 2208 (1997).

[25] V. Rodrigues, J. Bettini, A. R. Rocha, L. G. C. Rego, and D. Ugarte, Phys. Rev. B 65, 153402 (2002).

[26] A. Fujii, M. Tsutsui, S. Kurokawa, and A. Sakai, Phys. Rev. B 72, 045407 (2005).

[27] W. H. A. Thijssen, D. Marjenburgh, R. H. Bremmer, and J. M. van Ruitenbeek, Phys. Rev. Lett. 96, 026806 (2006).

[28] T. Kizuka, K. Yamada, S. Deguchi, M. Naruse, and N. Tanaka, Phys. Rev. B 55, R7398 (1997)

[29] T. Kizuka, Phys. Rev. Lett. 81, 4448 (1998).

[30] T. Kizuka, Phys. Rev. B 57, 11158 (1998).

[31] H. Ohnishi, Y. Kondo, and K. Takayanagi, Nature 395, 780 (1998).

[32] T. Kizuka, H. Ohmi, T. Sumi, K. Kumazawa, S. Deguchi, M. Naruse, S. Fujisawa, S. Sasaki, A. Yabe, and Y. Enomoto, Jpn. J. Appl. Phys. 40, L170 (2001).

[33] T. Kizuka, S. Umehara, and S. Fujisawa, Jpn. J. Appl. Phys. 40, L71 (2001).

[34] B. Ludoph and J. M. van Ruitenbeek, Phys. Rev. B 61, 2273 (2000).

[35] T. N. Todorov and A. P. Sutton, Phys. Rev. Lett. 70, 2138 (1993). 